\title{
Study of Testing on Transformers and its Significance
}

\author{
Ramesh Kumar Patel \\ M Tech Scholar \\ NRI Institute of Research \& Technology \\ Bhopal, (M.P) India \\ rameshpatel.cgl@gmail.com
}

\author{
Madhu Upadhyay \\ Head of Department \\ NRI Institute of Research \& Technology \\ Bhopal, (M.P) India \\ madyant44@gmail.com
}

\begin{abstract}
Transformers make up the main electrical network of the equipment. Failure of a transformer leads to loss of income and affects the reliability of the power supply for consumers. In order to ensure long and trouble-free operation of the transformers, various diagnostic tests are performed and corrective actions are initiated throughout their lifetime. There are many methods to access the residual life of transformers, for example short circuit tests on transformers and areas of application.
\end{abstract}

Keywords: Transformer, DGA, transformer health transformer failures, distribution transformer, real-time assessment

\section{INTRODUCTION}

Large power transformers are essential equipment for the power grid. Their reliability not only affects the availability of electricity in the supply area, but also affects the economic functioning of an energy supplier. Power transformer failures are problematic for four reasons: a transformer failure can cause system malfunction, in general large power transformers are placed in large flammable oil tanks and are harmful to the environment, utilities have no substitute in the event of maintenance or failure and ultimately the different types of thermal and electrical loads often age the transformer and cause it to fail. Therefore, utilities need to monitor the condition of the transformer, and also develop a diagnostic system. The insulating oil used in power transformers is a combination of liquid transformer oil and impregnated solid cellulose. Deterioration of the insulation and aging are the two main causes of the onset of the fault. The main causes of aging and deterioration of the insulation are thermal loads, electrical loads, mechanical loads and humidity. DGA approaches can assess the aging process and deterioration of the cellulosic material of the transformer oil, the degree of polymerization and furan analysis.

\section{LITERATURE REVIEW}

Xue Wang et al. [1] In the thesis, the overall stacking strategy is applied to fault diagnosis. The layered perceptron, nearest neighbor $\mathrm{k}$, decision tree, and support vector machine are used as learning components, and the random forest algorithm is used as a combined strategy to build a stacking diagnostic model. Furthermore, homogeneous ensemble methods are applied to the above four algorithms. The process uses the content of five characteristic gases as input parameters. Primary diagnostic results can be obtained with any basic classifier.

Edwell T. Mharakurwa et al. [2] in this article presented are the fuzzy logic method in the determining of the transformer failures and severity using the fault-forming energy of gases emitted during a transformer fault event. Furthermore, the energy of defect formation is a the dependent factor of the temperature for all the associated exhaust gases. The ADI energy-weighted are Instead, in the calculated total energy of the associated incipient error is used to determine the severity. The model results show that correctly identifying the type of fault and its severity based on the total energy released in the event of a fault can improve decision making by prioritizing the maintenance of failed transformers.

T. Kari, et al. [3] This paper presents another DGA technique for beginning determination of force transformer disappointments, in light of the versatile incorporated neuro-fluffy surmising framework (ANFIS) and the Dempster-Shafer hypothesis (DST). Four of the seven most well known ordinary techniques, considered, analyzed and thought about for better consistency and precision, are utilized to grow new ANFIS-based issue conclusion models. The presentation of the proposed strategy is contrasted and conventional methodologies and ANFIS-based models, which show that the proposed technique is better than different strategies and is more productive and stable for diagnosing power transformer disappointments with high exactness and consistency.

Yan Wang et al. [4] The article initially proposes another composite transformer issue conclusion technique that utilizes distinctive issue analysis models to play out the fundamental determination, then, at that point, utilizes the help transporter machine to play out the subsequent finding. The technique utilizes insightful correlative and combination believing that 
conquers the deficiencies of the single indicative model in diagnosing transformer disappointments and works on demonstrative precision and model reach.

G. K. Irungu et al. [5] this article presents the application of two of these techniques: fuzzy logic and reasoning. Over twenty case studies are evaluated with real field data, using the same inputs for both models. The data is viewed from different transformer perspectives, such as dissolved gases, physical, chemical and electrical properties of the insulation, but is ultimately classified into fault voltages and aging voltages. The results show that the evidence in terms of flexibility and ability to handle measurement uncertainties is better, while fuzzy logic is advantageous with a large amount of expert databases.

\section{Short Circuit AND OPEn Circuit Test ON TRANSFORMER}

This article presents the use of two of these techniques: fuzzy logic and reasoning. Over twenty case studies are evaluated with real field data, using the same inputs for both models. The data is viewed from different transformer perspectives, such as dissolved gases, physical, chemical and electrical properties of the insulation, but is ultimately broken down into fault voltages and aging voltages. The results show that the evidence for flexibility and handling of measurement uncertainties is better, while fuzzy logic is beneficial for a large number of experienced databases.

\section{A. Open Circuit Test}

The no-load test is used to determine the no-load current and losses of the transformer, thus determining its no-load parameters. This test is performed on the essential twisting of the transformer. Wattmeter, ammeter and voltage are associated with their essential winding. Their primary winding is powered by the rated rated voltage using the alternating current source.

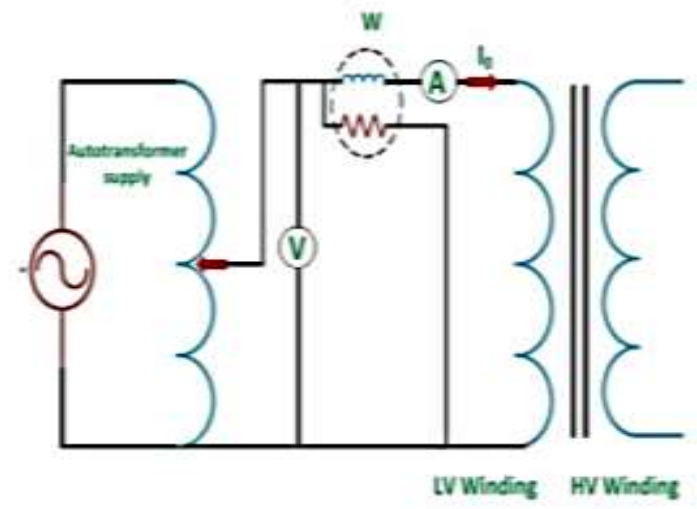

Fig. 3 open circuit test in the transformer Circuit Diagram
With the no-load test, the current of the non-load in losses of the transformer and therefore its no-load parameters are the determined. This test is performed on the essential twisting of the transformer. Wattmeter, ammeter and voltage are associated with their essential winding. Your primary winding is powered with rated voltage through the AC source.

\section{B. Short Circuit Test}

The short-circuit test is performed to determine the transformer parameters mentioned below.

Decides the copper loss that happens at full load. The copper loss is utilized to decide the productivity of the transformer.

The same opposition, impedance and spillage reactance are known from the short out test.

The short out test is performed on the auxiliary or high voltage twisting of the transformer. Estimating gadgets, for example, power meters, voltmeters and ammeters are associated with the high voltage twisting of the transformer. Its essential winding is shorted with thick tape or an ammeter associated with its terminal.

The low voltage source is changed to the auxiliary winding, which implies that the full burden current streams on both the optional and the essential side of the transformer. The full burden current is estimated by the ammeter associated with the auxiliary winding.

The wiring fig of the short-circuit test is shown on below:

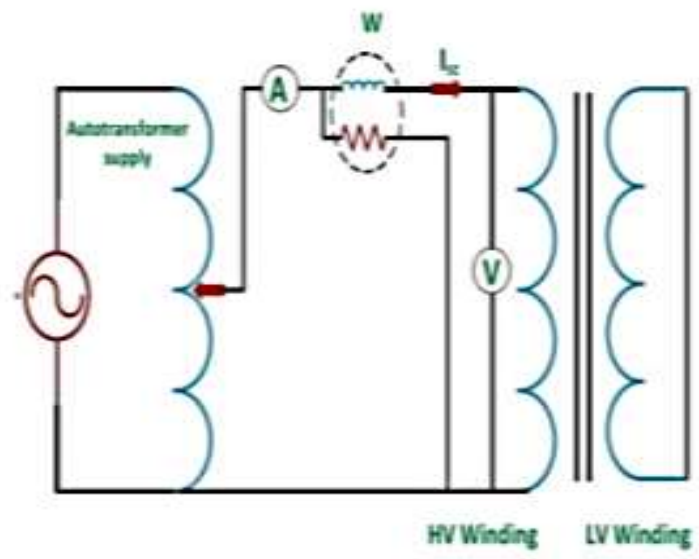

Fig. 4 Short Circuit Test on Transformer in Circuit Diagram

The low voltage source is applied across the auxiliary winding, which is around $5-10 \%$ of the ordinary appraised voltage. Flux 
builds up in the transformer core. The size of the river is small compared to the normal river.

The loss of iron from the transformer depends on the flow. Less occurs in the short circuit test due to the low flow rate. The power meter reading only determines the amount of copper loss that has occurred in their windings. The voltmeter estimates the voltage applied to its high voltage winding. Optional current is prompted in the transformer because of the applied voltage.

\section{ApPliCATIONS AREAS}

Current transformers for measurement and protection applications work essentially the same way: they convert highpower primary signals into readable secondary values. While current transformers for protection applications operate well above the rated current, current transformers for measurement purposes must go into saturation directly above the rated current to protect the connected measuring devices.

Protection current transformer. Current transformers play an important role in protecting electrical power systems. They provide the protection relay with a primary current ratio so that it can operate according to its settings.

The conversion of the current values from the primary to the secondary must be accurate under normal conditions and above all in fault conditions on the primary side (if currents up to 30 times the rated current are no exception).

Measure the current transformer. Energy is supplied from many sources, including alternative energy sources such as solar and wind power. Additional metering points are needed to ensure accurate billing in this competitive electricity market. It is important to calibrate the entire measurement chain because the meter is only as accurate as the transducers that power it. This makes testing and calibration of current transformers up to accuracy class 0.1 essential. However, field testing of 0.1 accuracy class current transformers is particularly critical, as interference from power lines can affect the measurement results.

\section{TESTING OF CURRENT TRANSFORMERS}

Conventional test methods apply a signal on one side and read the output signal on the other side.

There are several conventional test methods.

The traditional way to test a current transformer is to put a large amount of current on the primary and read the signals on the secondary. Using different loads or providing overcurrents, it is possible to simulate situations and measure and analyze signals on the secondary side. However, this process is lengthy and requires a lot of hardware. Sometimes this is not even possible because very high currents are required (for example, when testing current transformers designed for transient behavior (TP types) on site because they have very high knee point values).

Another common test scenario for current transformers is to introduce a defined test voltage on the secondary side and read the signals on the primary side. Unfortunately, some parameters such as accuracy and knee (arousal curve) can only be tested to a limited extent with this scenario. This is due to the accuracy limitations of the scenario due to the very weak signals used and the maximum voltage of about $2 \mathrm{kV}$ that can be applied to the secondary side of the current transformers. Other important parameters such as transient design factor, accuracy limit factor, safety factor, compound errors, time constants and many others cannot be tested at all.

O.C. and S.C. Tests are Calculation of the Efficiency

As we've seen, the applicable converter has two types of large losses, namely copper and core losses. These losses, which are dissipated in the form of heat, increase the temperature of the converter. Due to these losses, the input power consumed on the primary side no longer corresponds to the output power on the secondary side.

Therefore, the transfer efficiency is given as

Efficiency, $\eta=$ Power- $\mathrm{O} / \mathrm{P}$ in KW/ Power-I/P in KW

$=$ Power $\mathrm{O} / \mathrm{P}$ in KW/ $($ Power $\mathrm{O} / \mathrm{P}$ in KW + Losses)

$=$ Power $\mathrm{O} / \mathrm{P}$ in KW/ $($ Power $\mathrm{O} / \mathrm{P}$ in $\mathrm{KW}+$ Copper loss + Core loss)

We examined that the Pcore center misfortune stays steady from inactive to full load on the grounds that the transition in the center remaining parts consistent. Also, the copper misfortunes rely upon the square of the current. As the winding current differs from void to full load, the copper misfortunes likewise change.

Note that the transformer power KVA is $\mathrm{S}$, a load fraction $\mathrm{x}$ and the power of the factor load. And $\operatorname{Cos} \Phi$. Then

The $\mathrm{O} / \mathrm{P}$ power in $\mathrm{KW}=\mathrm{xSCos} \Phi$

Suppose the copper loss load are suppose is Pcu (since $\mathrm{x}=1$ ),

Then loss of copper at $\mathrm{x}$ per-unit loading $=\mathrm{x} 2 \mathrm{Pcu}$

Therefore the transformer efficiency are 


$$
\text { Efficiency, } \eta=x S C o s \Phi /(x S \operatorname{Cos} \Phi+x 2 \text { Pxcu }+ \text { Pxcore })
$$

Above $\mathrm{eq}^{\mathrm{n}}$ in show of the efficiency, present in the core or iron losses and full load copper losses are the determine by the open circuit test and short circuit test.

\section{CONCLUSION}

The role of the manufacturer in designing the transformer for a particular application is very important. A detailed review of the design calculations for various parameters, including sufficient design leeway for strong electromagnetic forces during a short circuit, is very important before production begins. The international standard IEC 60076-5 prescribes short-circuit tests to demonstrate the dynamic short-circuit resistance of power transformers. According to the standard, the short-circuit reactance of the transformer must be measured before and after each short-circuit. From the modification of the winding geometry, conclusions can be drawn about the value of the variation of the inductive reactance due to the dynamic effect of the short-circuit current. It is therefore a very important electrical parameter of the device to be tested in relation to the qualification of the transformer, therefore the standard limits the value of the variation of the inductive reactance.

\section{REFERENCES}

[1] X Wang, T. Han "Transformer Fault Diagnosis Based on Stacking Ensemble Learning"2020. https://doi.org/10.1002/tee.23247, 02 October 2020.

Edwell T.Mharakurwa, G.N.Nyakoe "Power Transformer Fault Severity Estimation Based on Dissolved Gas Analysis and Energy of Fault Formation Technique" in Journal of Electrical and Computer Engineering2019.

T.Kari, W.Gao, D. Zhao, "An integrated method of ANFIS and Dempster-Shafer theory for fault diagnosis of power transformer," IEEE Transactions onDielectrics and Electrical Insulation, vol.25, no.1, pp.360-371,2018.

[4] Y. Wang,Liguo Zhang“A Combined Fault Diagnosis Method for Power Transformer in Big Data Environment" Mathematical Problems in Engineering2017

O.E. Gouda, S.M. Saleh, and S.H. El-hoshy,"Power transformer incipient faults diagnosis based on dissolved gas analysis," Indonesian Journal of Electrical Engineering and Computer Science, vol.1, no.1, pp.10-16,2016.

G.K. Irungu, A.O. Akumu, and J.L. Munda, "Application of fuzzy logic and evidential reasoning methodologies in transformer insulation stress assessment,"IEEE Transactions on Dielectrics and Electrical Insulation, vol.23, no.3, pp. 1444-1452,2016. remnant life prediction and asset management decision," in the

IEEETransactions on Dielectrics and Electrical Insulation, vol23, no.5, pp.3199-3208, 2016.

Wang, N.; Zhao, F. An Assessment of the Condition of Distribution Network Equipment Based on Large Data Fuzzy Decision-Making. Energies 2020, 13, 197.

Tran,Q.T.Davies, K.;Roose, L.Machine learning for assessing the service transformer health using an energy monitor deviceIOSR J. Electr. Electron. Eng.2020, 15,1-6 